\title{
Transformaciones en el mercado de trabajo de universitarios en América Latina: los impulsos al inicio de la fijación de salario mínimo profesional
}

Changes in the labor market of university in Latin America: the pulses at the start of fixing minimum professional wage As mudanças no mercado de trabalho de universitários
na América Latina: os impulsos para início da fixação do
salário mínimo profissional Claudio Rama ${ }^{[a, b]^{*}}$

[a] Universidad de la Empresa (UDE), Facultad de Ciencias Empresariales, Montevideo, Uruguay [b] Sistema Nacional de Investigadores (SIN/ANII), Montevideo, Uruguay

\section{Resumen}

El artículo presenta la existencia de una nueva dinámica en los mercados de trabajos profesionales, en América Latina, como resultado del aumento de la cobertura y de la eficiencia

* CR: doctor en Educación (UNSR), doctor en Derecho (UBA), postdoctorado (UNESR, UNICAMP, UFF, UBA), e-mail: claudiorama@gmail.com 
de la graduación de estudiantes. Detalla los diversos impactos del egreso universitario en los distintos mercados profesionales. Entre esos impactos muestra la irrupción de una tendencia a la fijación del salario mínimo profesional. Concibe a los colegios profesionales como actores en esta regulación salarial diferenciada en función de los marcos normativos de cada país en relación a su rol en la regulación del ejercicio profesional. El artículo muestra una alta diferenciación en la regulación por parte de los colegios profesionales en la región y visualiza a la vez el inicio de políticas de carácter nacional de fijación del salario mínimo de los universitarios. Finalmente sostiene que la discusión sobre el salario mínimo profesional será uno de los ejes de los debates académicos y de la política pública en materia de la regulación universitaria.

Palabras Clave: Salarios profesionales. Mercados laborales. Egreso profesional. Educación Superior. América Latina.

\begin{abstract}
The article shows the existence of a new dynamic in the markets for professional work in Latin America, as a result of increased coverage and efficiency of student graduation. Details the various impacts of university graduation in various professional markets. Among these impacts shows the arrival of a trend-setting professional minimum wage. Conceives professional associations as actors in this wage differential regulation based on the regulatory frameworks of each country in relation to its role in regulating the profession. The article shows a high differentiation in the regulation by the professional bodies in the region and displays both the start of policies at the national level for fixing the minimum wage for college. Finally he argues that the discussion about the professional minimum wage will be one of the pillars of academic debates and public policy of the university regulation.
\end{abstract}

Keywords: Professional salaries. Labor markets. Professional graduation. Higher Education, Latin America.

\title{
Resumo
}

O artigo mostra a existência de uma nova dinâmica nos mercados de trabalho profissionais, na América Latina, como resultado do aumento da cobertura e da eficiência da graduação de estudantes. Ele detalha os vários impactos da graduação universitária em vários mercados 
profissionais. Esses impactos mostram o surgimento de uma tendência à determinação de um salário mínimo profissional. Concebe associações profissionais como atores dessa regulação do diferencial de salário, dependendo dos marcos normativos de cada país em relação a seu papel na regulação da profissão. $O$ artigo revela uma elevada diferença entre organizações profissionais da região no que diz respeito à regulação e mostra, ao mesmo tempo, o início de políticas, em nível nacional, para a fixação de salários mínimos para universitários. Finalmente argumenta que a discussão sobre o salário mínimo profissional será um dos eixos do debate acadêmico e das políticas públicas sobre a regulamentação da universidade.

Palavras-chave: Salários profissionais. Mercado de trabalho. Educação superior. América Latina.

\section{El aumento del egreso de profesionales universitarios en América Latina}

Históricamente los salarios de los profesionales han sido superiores a los salarios de las personas sin formación en la región ${ }^{1}$. Ello ha sido resultado de que los retornos en educación terciaria son superiores en los países en desarrollo por la escasez permanente de capital humano especializado (PSACHAROPOULOS, 1980, 1994). En la década del 1990, tal brecha de desequilibrios en los mercados de trabajo y educación se incrementó y los ingresos de los trabajadores profesionales con título aumentaron más que los salarios de las personas sin formación especializada (WELLER, 2004) como resultado de una apertura, del cambio tecnológico y del inicio de una transformación en el padrón de acumulación

1 Ha habido muchos estudios en la materia. Sin embargo, a pesar de haberse comprobado exhaustivamente que la inversión en capital humano a través de una mayor educación formal es un determinante importante de los ingresos de los jefes de hogar, en un estudio de México se verificó también que los factores de carácter territorial y social, así como el tamaño del establecimiento y el tipo de actividad económica son relevantes en la determinación de los ingresos de los jefes de hogar (VARELA; URCIAGA, 2012). Igualmente impactan la calidad de la institución, el género y el tipo de mercado laboral. 
de capital que incrementó la demanda de trabajo con mayor capacitación. Sin embargo, a medida que está aumentando el egreso universitario, las dinámicas de los mercados laborales muestran en la década del 2000 que los diferenciales salariales en algunos países de la región no parecen estarse ensanchando en igual dimensión que en el pasado. Ello respondería además a cambios en las políticas públicas con un persistente aumento del salario mínimo que ha reducido la tasa de retorno profesional comparativamente. Asociado a ello se asiste a un aumento del desempleo profesional y de la emigración. Es claro que los diferenciales salariales son estructurales y se asocian al impacto del aumento de la formación universitaria en el incremento de la productividad, el cual deriva - aunque en diversa proporción en función de los niveles de desempleo y competencia así como de la calidad de las instituciones - en un aumento de los salarios y menores niveles de desempleo.

Así - salarios y empleo - son los componentes que han retroalimentado el ciclo de aumento continuo de la matrícula universitaria y la propensión a la inversión en educación superior por parte de las familias, y que se soporta en la teoría del capital humano que plantea que la educación es una inversión que genera beneficios salariales asociados a la productividad de las competencias profesionales adquiridas. Sin embargo, este marco conceptual se concibe como un modelo simple de causa efecto, y en el cual el nivel de remuneración de los mercados y los costos directos e indirectos educativos establecen la tasa interna de retorno de la inversión en formación. En este escenario la determinación de los salarios es afectada directamente por el aumento de la cobertura y los egresos por aumento de la eficiencia y diferenciación de las instituciones, la mayor articulación educación mercado, expansión privada, feminización, flexibilización de las formas de titulación y reducción de los tiempos de estudios del grado.

En los últimos años se ha producido en América Latina un cambio radical en la dinámica universitaria y de los mercados laborales gracias al incremento de la cobertura y el aumento del egreso profesional. La relativa histórica carencia de profesionales está cambiando significativamente a medida que aumenta la oferta de egresados universitarios (RAMA, 
2014). Para el 2010 el total de egresados profesionales de la región fue de 2:073.007, de los cuales el 91,3\% egreso de estudios de grado y el resto en maestrías y doctorado (Tabla 1).

Tabla 1 - Egreso de profesionales en América Latina 2010

\begin{tabular}{lcccccc}
\hline País & Año & $\begin{array}{c}\text { Egreso } \\
\text { Grado }\end{array}$ & Años & $\begin{array}{c}\text { Egreso } \\
\text { Maestría }\end{array}$ & Año & $\begin{array}{c}\text { Egreso } \\
\text { doctorado }\end{array}$ \\
\hline Argentina & 2010 & 99431 & 2010 & 2962 & 2010 & 1504 \\
Bolivia & 2010 & 25020 & 2010 & 2264 & 2002 & 10 \\
Brasil & 2010 & 829286 & 2010 & 39590 & 2010 & 11314 \\
Chile & 2010 & 64229 & 2010 & 7698 & 2010 & 433 \\
Colombia & 2010 & 111404 & 2010 & 5902 & 2010 & 211 \\
Costa Rica & 2010 & 38163 & 2010 & 4443 & 2010 & 117 \\
Cuba & 2010 & 84779 & SD & SD & 2010 & 617 \\
Ecuador & 2008 & 48911 & 2008 & 4570 & 2008 & 53 \\
Guatemala & 2010 & 10700 & 2010 & 2212 & 2010 & 16 \\
Honduras & 2006 & 9293 & 2006 & 375 & SD & SD \\
Jamaica & 1997 & 2865 & 2011 & 276 & 2011 & 18 \\
México & 2010 & 344651 & 2010 & 44318 & 2010 & 4167 \\
Nicaragua & 2004 & 7713 & 2004 & 527 & 2004 & 111 \\
Panamá & 2003 & 13169 & 2003 & 711 & 2003 & 5 \\
Perú & 2008 & 47895 & 2010 & 5009 & 2010 & 786 \\
Paraguay & 2008 & 5594 & 2008 & 1013 & 2011 & 164 \\
El Salvador & 2010 & 17089 & 2010 & 490 & 2010 & 2 \\
Trin. y Toba & 2010 & 2517 & 2010 & 451 & 2010 & 19 \\
Uruguay & 2010 & 5450 & 2010 & 649 & 2010 & 39 \\
Venezuela & 2009 & 124706 & 2008 & 3296 & 2007 & 335 \\
\hline América & & 1892865 & & 160221 & & 19921 \\
Latina & $100 \%$ & $91,3 \%$ & & $18,1 \%$ & & $4,06 \%$ \\
\hline \% & & & & & & \\
\hline & & & & & & \\
\hline
\end{tabular}

Fuente: RICYT, 2015. 
Esta situación es resultado de una evolución continua y sistemática de los egresos profesionales (Tabla 2), que muestran tasas significativas resultado de múltiples variables entre las cuales el aumento de los recursos económicos de los hogares que reduce la deserción y aumenta la eficiencia de titulación, procesos educativos más flexibles y con mayores niveles de regionalización que permiten el ingreso y el egreso de mas estudiantes de los sectores con menos niveles de egreso como los trabajadores y las personas del interior. El aumento de la titulación, más allá de la masificación de la matrícula, es incentivado a su vez por una tendencia a la reducción de los años de estudio y el aumento de los estudios técnicos. Estudios nocturnos y un mayor peso de la educación privada que tienen tasas de titulación superiores también han contribuido a ello. Hasta la feminización ha facilitado el aumento del egreso resultado de una mayor titulación de las mujeres. Este es un proceso que no para de crecer como resultado de nuevas dinámicas educativas y que está implicando un cambio en los mercados laborales. El aumento de la matricula en educación a distancia en la región también está incidiendo en mayores niveles de egreso. En Brasil, por ejemplo, para el 2012 la cobertura de la educación superior alcanzó a 15,8\% de la matricula, siendo entre los nuevos estudiantes el 31\% (HOPER EDUCAÇÃO, 2014).

Tabla 2 - Crecimiento de los egresos profesionales de grado en algunos países (1990-2010)

(continúa)

\begin{tabular}{lcccccc}
\hline & 1990 & 2000 & $\mathbf{2 0 1 0}$ & $\begin{array}{c}\text { \% Aumento } \\
\text { Anual } \\
1990-2000\end{array}$ & $\begin{array}{c}\text { \% Aumento } \\
\text { Anual } \\
\mathbf{2 0 0 0 - 2 0 1 0}\end{array}$ & $\begin{array}{c}\text { \% Aumento } \\
\text { Anual } \\
\text { 1990/2010 }\end{array}$ \\
\hline Argentina & 33465 & 62473 & 99431 & 6,4 & 4,8 & 5,6 \\
Bolivia & 2731 & 6967 & 25020 & 9,8 & 14 & 11,7 \\
Brasil & & 348653 & 829286 & & 9 & \\
Chile & 12401 & & 64229 & & & 8,57 \\
Colombia & 50188 & 77923 & 111404 & 4,5 & 3,6 & 4,1 \\
\hline
\end{tabular}


Tabla 2 - Crecimiento de los egresos profesionales de grado en algunos países (1990-2010)

(conclusión)

\begin{tabular}{|c|c|c|c|c|c|c|}
\hline & 1990 & 2000 & 2010 & $\begin{array}{c}\text { \% Aumento } \\
\text { Anual } \\
1990-2000\end{array}$ & $\begin{array}{c}\% \text { Aumento } \\
\text { Anual } \\
2000-2010\end{array}$ & $\begin{array}{c}\text { \% Aumento } \\
\text { Anual } \\
1990 / 2010\end{array}$ \\
\hline Costa Rica & 1681 & 26469 & 38163 & 31,7 & 3,7 & 16,9 \\
\hline Cuba & 35144 & 16496 & 84779 & $-7,3$ & 17,8 & 4,5 \\
\hline Guatemala & & 2336 & 10700 & & 16,4 & \\
\hline Honduras & 768 & 7503 & & 25,6 & & \\
\hline Jamaica & 1770 & & & & & \\
\hline México & 118424 & 209795 & 344651 & 5,9 & 5,1 & 5,5 \\
\hline Panamá & 2655 & 13852 & & 18,0 & & \\
\hline Perú & 14722 & 38453 & & 10,1 & & \\
\hline Paraguay & 1361 & 1940 & & 3,6 & & \\
\hline El Salvador & & 11031 & 17089 & & 4,5 & \\
\hline Tri. y Tob & 628 & 1071 & 2517 & 5,5 & 8,9 & 7,2 \\
\hline Uruguay & 1790 & 3574 & 5450 & 7,2 & 4,3 & 5,7 \\
\hline
\end{tabular}

Fuente: RICYT, 2015.

\section{Los impactos en los mercados laborales de profesionales universitarios en América Latina}

El crecimiento del egreso en todos los países ha sido superior al aumento de las economías nacionales y de la población económicamente activa, cambiando la composición de los mercados de trabajo. Tal egreso ha sido relativamente absorbido en las últimas décadas, gracias a la modernización de la estructura productiva asociada a la apertura, el cambio tecnológico y la expansión de modelos de exportación primarios con mayor valor agregado por incorporación de más tecnologías gracias al aumento de los precios internacionales. Ello se expresó en un aumento continuo de la proporción de profesionales en la población económicamente activa (PEA) que ya oscila entre el $8 \%$ y el $19 \%$. Sobre la población 
de más de 25 años en algunos países en algunos países anda en el entorno de entre 20 y 30\%. En Costa Rica por ejemplo, es $24 \%$.

La incorporación de tecnologías en el proceso económico asociado a la mayor apertura, impacta en la demanda de trabajadores calificados y profesionales (CARNOY, 2006). Desde los 1990, la apertura económica incrementó la competencia en los distintos mercados y más inversiones en tecnológicas han redundado en mayor empleo profesional. Sin embargo, el nivel del egreso profesional ha sido superior y ello ha derivado en una complejización de estos mercados laborales con un aumento del componente de profesionales en la migración, un crecimiento del empleo profesional en puestos previamente ocupados por técnicos o administrativos, un retraso en el ingreso al mercado de trabajo mediante una continuación de la formación a través de la realización de estudios de postgrados, la caída relativa de los salarios de los profesionales y sobre todo de aquellos en instituciones y programas no acreditados, así como un aumento del nivel de desempleo de los profesionales y una reducción de la diferencia salarial entre hombres y mujeres.

No existe un equilibrio general entre oferta y demanda de profesionales sino que ambos tienen sus propias determinaciones independientes, y el crecimiento de la matrícula y del egreso a escala regional tiende a ser superior a la demanda local de empleo, derivando en una tendencia estructural hacia la sobreoferta y a la sobre calificación de educación superior. Esta es, a su vez, facilitada por las propias características de los desequilibrios estructurales de los mercados de oferta y demanda, como por la existencia de mercados internacionales de colocación de excedentes de personal profesional. La Organización para la Cooperación y el Desarrollo Económicos (OCDE) ha analizado estos procesos a escala global como derivación de cambios en los perfiles demográficos en los países centrales y la demanda de profesionales externos (OCDE, 2012). La tendencia a la sobre oferta profesional y por ende a la sobre calificación, ha sido favorecida por la gratuidad y el incremento de la competencia entre los profesionales, se está expresando además en la emigración profesional. Esta se está conformando en un componente estructural de los 
mercados profesionales. Los niveles de desempleo profesional y las tasas de retorno, junto con la demanda externa y las posibilidades de trabajo, se constituyen en impulsores de la emigración profesional.

La sobre oferta y la sobre calificación se constituyen en expresiones de los desequilibrios y también en las formas de funcionamiento de esos mercados laborales. Sin embargo también al tiempo actúan como mecanismos para acotar los aumentos salariales reconstruyendo un nivel de equilibrio entre oferta y demanda de trabajo profesional. Igualmente, la sobre oferta y sobre calificación facilita la movilidad profesional ante mercados internacionales que demandan crecientemente profesionales y técnicos calificados.

Analizaremos a continuación algunos de los impactos de las dinámicas producidas por el aumento del egreso profesionales sobre estos mercados laborales y educativos.

\section{Incorporación en el mercado formal en puestos de perfil profesional}

Los cambios productivos con la modernización tecnológica están promoviendo la expansión del sector formal de las economías con un mayor componente del trabajo profesional. El ejercicio profesional se basa en una lógica de diagnóstico y respuesta y para ello se requiere más información y competencias. Hay entonces una directa relación entre su trabajo y el uso de equipos y aplicaciones informáticas, que impone un tipo de empleo profesional asociado a la informática. El empleo profesional está en estrecha correlación con la expansión del sector servicios, del empleo en el Estado y en áreas modernas de exportación. La oferta y el empleo profesional es altamente diferenciada por profesiones $\mathrm{y}$ lentamente el mercado tenderá a absorber con mayor intensidad a los profesionales provenientes de instituciones con certificaciones acreditadas como forma de asignar puestos de trabajo en un escenario competitivo. El Observatorio del Mercado Laboral en Colombia muestra que los 
egresados de los programas acreditados tienden a recibir mayores niveles de remuneración.

\section{Sustitución de puestos de trabajos no profesionales}

La sobreoferta de profesionales deriva en su incorporación en mercados de trabajo que antes eran ocupados por personas de menor formación. Siguiendo el "efecto fila", en función de las certificaciones y de su calidad se produce una sustitución de empleos por personas sin certificación por profesionales a los mismos salarios. El ingreso de personal técnico y profesional o inclusive de desertantes universitarios en esos puestos, promueve un mejoramiento de la productividad. Ello cambia los mercados laborales mediante la renovación de los puestos de trabajo. Impacta también en los patrones de emigración de trabajadores de baja formación y en los componentes del desempleo. La sustitución de puestos técnicos o administrativos por profesionales, se asocia en muchos casos a certificaciones de baja calidad, de instituciones o programas no acreditados o a distancia, o en campos profesionales con alta saturación de oferta de egresados.

\section{Aumento del desempleo profesional}

El aumento de los egresados profesionales y técnicos en América Latina tiende a ser superior a su absorción tanto en los mercados formales como informales, creándose una tendencia de largo plazo al aumento del desempleo profesional. Este se constituye en un ejército de reserva laboral profesional que impacta en los salarios y en la emigración profesional. Al tiempo impacta inversamente sobre esta tasa de desempleo de otros sectores de menor formación. Al aumentar el desempleo profesional la tasa de retorno se reduce ya que disminuye a la vez la probabilidad de conseguir empleo (ADROGUÉ, 2006). El nivel de desempleo profesional es sin embargo altamente diferenciado por países asociado a la distinta composición 
de los mercados de trabajo profesionales y a los niveles de saturación y diferenciación de los diversos mercados profesionales. Así, los niveles de desempleo de los profesionales tienen múltiples determinantes, destacándose el área de conocimientos, la realidad económica de los mercados, el tipo de institución de donde se proviene, el género, e inclusive la familia y origen social de los egresados (VRIES; NAVARRO, 2011). Sin embargo a escala regional se aprecia un crecimiento constante y continuo de los niveles del desempleo en las últimas dos décadas que ha llevado el desempleo del 4,69\% en 1990 al 6,63\% en el 2010. De punta a punta hay un 40\% de aumento del desempleo en 20 años como se observa en la Tabla 3.

Tabla 3 - Desempleo profesional en América Latina (1990-2010)

\begin{tabular}{ccccccccccc}
\hline Año & 1990 & 1991 & 1993 & 1994 & 1995 & 1996 & 1997 & 1998 & 1999 & 2000 \\
\hline $\begin{array}{l}\text { \% desempleo } \\
\text { profesional }\end{array}$ & 4,69 & 11,3 & 8,45 & 5,16 & 4,9 & 4 & 7,01 & 6,25 & 7,53 & 4,35 \\
\hline Año & 2001 & 2002 & 2003 & 2004 & 2005 & 2006 & 2007 & 2008 & 2009 & 2010 \\
\hline $\begin{array}{l}\text { \% desempleo } \\
\text { profesional }\end{array}$ & 6,192 & 8,88 & 9,13 & 8,11 & 7,58 & 5,65 & 5,91 & 5,85 & 6,58 & 6,63 \\
\hline
\end{tabular}

Fuente: CEPAL; OIT, 2015; OIT, 2013.

\section{Propensión a la emigración profesional}

Aunque el impacto más significativo del aumento del egreso es el empleo profesional, también se constata un aumento de la propensión a la emigración profesional. Hay un incremento de la proporción de profesionales dentro de la masa emigrante en varios países. Tal emigración se da con más intensidad en los países en los cuales el desempleo profesional es más alto, en aquellos en los cuales la tasa de retorno profesional es relativamente más baja comparativamente a nivel internacional, en los países donde el crecimiento del producto es menor y donde el egreso profesional es mayor. En muchos casos, los emigrantes profesionales 
tienden a ser aquellos que no logran insertarse en los mercados laborales locales. Otros motivos como los políticos tienen también su impacto en el caso de Venezuela o de seguridad en el caso de México. En Brasil, México, Argentina, Venezuela, Uruguay o República Dominicana se constata un aumento de la emigración de profesionales y técnicos universitarios que se conforma como una corriente de exportación y un drenaje continuo de capital humano. Los datos de las visas de profesionales en Estados Unidos muestran su correlación con los aumentos de los egresos en la región (ARAGONÉS; SALGADO, 2011).

Los desequilibrios de los mercados educativos locales se tienden a ajustar en el marco de un nuevo contexto económico mundial que valoriza más intensamente los recursos altamente capacitados y que presiona por un aumento de la emigración profesional. Hay un patrón migratorio global que se está profundizando y desde la crisis del 2009 se expresa en la expulsión de los antiguos emigrantes de baja calificación y a la par en un aumento de la migración selectiva capacitada (ARAGONÉS, 2011).

\section{Ingreso tardío al mercado de trabajo y continuación de los estudios}

El aumento de la formación de capital mediante estudios de postgrado se constituye en una de las expresiones de los desequilibrios entre la educación y el trabajo. La sobre oferta de profesionales de grado impulsa la sobre calificación en un contexto de mayor especialización de las demandas laborales. Tal aumento de la formación de capital humano se entronca con la internacionalización educativa para adquirir dichas competencias. Los postgrados se constituyen en una educación internacional en tanto a escala nacional no se pueden ofrecer todas las especializaciones disciplinarias y expresan los conocimientos de la creciente sociedad de la información (RAMA, 2007). Además de los estudios de postgrado a escala local, asistimos a una demanda por estudios de postgrado internacionales. 
Estudios de seguimiento de egresados muestran una mayor propensión a continuar estudiando asociado a los niveles competitivos de las profesiones, a la duración de los estudios de grado, y a las tasas de retorno diferenciadas de ambos mercados (grado y postgrado y al interior de estos) (FORERO; RAMÍREZ, 2008).

El postgrado también se constituye como un mecanismo asociado a la emigración en tanto constituye un mecanismo de selección de ingreso a los mercados laborales externos y una estrategia personal de sobrevivencia a través de la emigración profesional. Ello se expresa en la alta participación de los estudios en el extranjero en el total de postgrados de la región y en el dato de que hasta el $20 \%$ de los que terminan los estudios en el extranjero no regresan a sus países de origen. El carácter gratuito de los estudios públicos facilita esta dinámica, más allá de la participación de egresados de instituciones privadas, que permite mostrar que hay una prima positiva salarial para ambos tipos de egresados.

\section{Los salarios de los profesionales}

El mayor salario de los profesionales y las menores tasas de desempleo constituyen motores importantes en la demanda de la formación profesional y el sacrificio de rentas y costos de oportunidad para dedicarse a la formación. Ello, junto a otras causas, ha marcado el aumento de la cobertura y del egreso. Tal dinámica, de oferta ante una demanda más rezagada, impacta en los mercados profesionales y en los salarios. Estamos frente a una dinámica a escala global que deriva en una caída de la tasa de retorno de la inversión educativa. La reducción del salario relativo resultado de ese cambio entre la oferta y demanda, constituye un desestímulo a los estudios e impacta negativamente a través de las externalidades en las capacidades de la sociedad. La gratuidad del sector público en la región ha impulsado a las personas de ingresos menores a destinar tiempo y esfuerzo a su formación con miras a dotar a la sociedad de los profesionales requeridos. Ahora, la caída de los retornos replantea el problema desde el 
ángulo del mercado de trabajo. Tal tendencia de largo plazo lleva al análisis de la mayor regulación del mercado de trabajo profesional mediante un mayor control de las certificaciones, de mínimos de formación e inclusive de establecimiento de un salario mínimo profesional.

\section{El salario mínimo de los trabajadores manuales}

El establecimiento del salario mínimo de los trabajadores fue establecido en Australia en 1894 y se fue generalizando a todos los países. Esta regulación de salario y el establecimiento de la jornada laboral de 40 horas, fijaron los límites de la relación contractual con el trabajo en el marco de la industrialización y la conformación de las sociedades contemporáneas. El salario mínimo constituyó una regulación de política pública que fijó la dinámica de la competencia entre las empresas y el funcionamiento del mercado de trabajo. Fue además el inicio de una regulación de los mercados de trabajo en términos de las prestaciones sociales, las condiciones del trabajo, las licencias, la responsabilidad, los deberes y derechos de las partes y así sucesivamente.

El establecimiento del salario mínimo se basó en el concepto de un mínimo salarial que permitiese la continuación de la reproducción de la fuerza de trabajo en condiciones que garantizaran la calidad del trabajo. El salario mínimo se vinculó a los ingresos necesarios para mantener las condiciones mínimas, que en general se asociaban a la alimentación, la salud y la vivienda. Probablemente expresó también una dinámica donde la oferta de mano de obra superaba a la demanda, lo cual podía impulsar niveles salariales menores, y donde la política imponía condiciones de salarios mínimos para garantizar la capacidad de supervivencia del propio trabajo en el mediano y largo plazo.

El salario mínimo fue una conquista social más allá de su utilidad como factor regulador de la dotación continua de los mercados. Cumplió además el ser un mecanismo que facilitó las migraciones del campo a la ciudad y la constitución de bolsones de oferta de mano de obra 
y la constitución de mayores contingentes de fuerza de trabajo que permitiera la dinámica de crecimiento económico. También organizó la dinámica de relación en el campo del trabajo y el salario mínimo se constituyó a su vez en la base de las negociaciones colectivas y en el marco central de la regulación de los mercados de trabajo, fundamentalmente en aquellos lugares donde no existían negociaciones salariales en las empresas. El salario mínimo actualmente en la mayor parte de los países del mundo emana del gobierno y regula a una parte del mercado laboral, no solamente obrero, e impacta fundamentalmente a los trabajadores no sindicalizados o sin contratos especiales. Es a la vez altamente diferenciado a escala regional con dimensiones que van desde 160 dólares en Nicaragua a 516 en Costa Rica. Los niveles de devaluación y de existencia de dólares paralelos complejiza la paridad del poder de compra y los niveles comparativos, y el grado de formalización de los mercados de trabajo determina su grado de generalización.

\section{El salario mínimo del trabajo profesional}

La expansión de la matrícula y del egreso universitario al introducir cambios en su incidencia en la población económicamente activa ha comenzado a plantear la necesidad de encarar el salario mínimo profesional con miras a garantizar la formación. Esta reflexión se plantea ante una dinámica de mayor oferta frente a la demanda y de caída de caída de los retornos profesionales. Estos a su vez, están a su vez impulsando una mayor gratuidad pública y dificultades de captación de matrícula privada que al tiempo se encarece por las demandas de calidad.

La introducción de un salario mínimo profesional tiene sin embargo fuertes limitaciones y complejidades de instrumentación. Por una parte la regulación del ejercicio profesional está altamente fragmentada y en muchos países privan concepciones constitucionales asociadas a la libertad del ejercicio profesional. Igualmente hay una carencia de organismos gremiales de profesionales y una alta diversidad de campos y tareas 
profesionales. El ejercicio profesional además aunque tiene una mayor incidencia en régimen de dependencia, mantiene en algunas profesiones un alto peso del ejercicio profesional independiente.

La regulación del ejercicio profesional es altamente diferenciada en América Latina. Mientras que en algunos países de la región ello recae en los organismos gremiales, en otros es mandato del Gobierno central, en tanto que en oros descansa en los segundos niveles del Estado, en los Estados, Departamentos o Provincias. Sin embargo, a pesar de las limitaciones, las demandas de una regulación salarial sobre estos mercados han ido desarrollando diversas iniciativas y acciones para su establecimiento (Cuadro 1).

Cuadro 1 - La colegialización profesional en América Latina: análisis de casos nacionales

(continúa)

Engeneral es obligatorio por Leyes específicas el registro del
título en el Colegio profesional respectivo como requisito para el
ejercicio en varias profesiones. Tal registro o matrícula, a nivel de
las asociaciones profesionales se realiza a nivel de las Provincias que
son las que constitucionalmente tienen el mandato de supervisión
de las profesiones. El requisito de la Colegiación obligatoria por Ley
provincial requiere el grado académico requerido y el pago de la cuota
que anualmente fije el Colegio respectivo autónomamente. Hay leyes
que han delegado el poder regulador del Estado a nivel provincial a
los Colegios profesionales. El nivel Federal de regulación profesional
históricamente ha venido decreciendo. La certificación y recertificación
puede ser una actividad a cargo de las Universidades, o de los Colegios
o las Asociaciones Profesionales, en tanto la Ley de Educación Superior
(1995) habilitó ambas posibilidades.
Los títulos son reconocidos por el Ministerio de Educación con el
objeto de darles validez nacional a partir de acuerdos del Consejo
de Educación Federal. Las Universidades realizan la certificación de
los títulos de especialistas de los estudios realizados por Sociedades
Científicas (medicina). En algunos casos las propias Sociedades realizan
las certificaciones.
Los títulos de las Universidades públicas no requieren licenciamiento,
pero los de las Universidades privadas requieren una “Provisión
Nacional” otorgada por el Ministerio de Educación y Cultura a nombre
del Estado, que funciona como certificación pública que da validez al
título. Es obligatorio pertenecer al Colegio Profesional respectivo para
poder ejercer la profesión en varias profesiones a partir de la existencia
de leyes específicas que lo han establecido.

Rev. Diálogo Educ., Curitiba, v. 15, n. 45, p. 597-623, maio/ago. 2015 


\section{Cuadro 1 - La colegialización profesional en América Latina: análisis de casos nacionales}

(continúa)

\begin{tabular}{|c|c|}
\hline Bolivia & $\begin{array}{l}\text { La inscripción es Departamental (9) y es parte de un sistema nacional } \\
\text { de Colegios Profesionales que se unifica a nivel de cada Departamento } \\
\text { conformando la Federación Departamental de Profesionales. Las } \\
\text { nueve Federaciones Departamentales y conforman la Confederación } \\
\text { Nacional de Profesionales de Bolivia. Por Ley, los abogados para poder } \\
\text { matricularse además de los requisitos académicos deben prestar un } \\
\text { "juramento profesional" que es recibido en audiencia pública en forma } \\
\text { individual por la Suprema Corte y el Colegio de Abogados. Actualmente } \\
\text { se ha iniciado una política pública de desregulación del control de los } \\
\text { Colegios. En la profesión de abogado ya no es obligatoria la colegiación } \\
\text { sino simplemente el juramento ante la Suprema Corte. }\end{array}$ \\
\hline Brasil & $\begin{array}{l}\text { Los títulos universitarios tienen validez nacional legalmente y son } \\
\text { registrados por las propias Universidades. Los títulos conferidos por } \\
\text { las instituciones no universitarias (Facultades, Centros universitarios) } \\
\text { deben registrarse en las Universidades indicadas por el Consejo } \\
\text { Nacional de Educación. Los Colegios Profesionales certifican los títulos } \\
\text { y por Ley de cada profesión la inscripción es obligatoria. La inscripción } \\
\text { en los Colegios es en cada Estado y no se puede ejercer en otro Estado } \\
\text { sin inscribirse en el Colegio de ese Estado, el cual fija los requisitos } \\
\text { propios de inscripción y por lo tanto de ejercicio laboral. Hay una fuerte } \\
\text { tendencia as la delegación por ley de la fiscalización de las profesiones } \\
\text { reguladas a los Colegios profesionales. Los Consejos Profesionales fijan } \\
\text { los requisitos de colegialización en cada caso. }\end{array}$ \\
\hline Chile & $\begin{array}{l}\text { La Constitución de } 1980 \text { establece que "ninguna ley o disposición de } \\
\text { autoridad pública podrá exigir la afiliación a organización o entidad } \\
\text { alguna como requisito para desarrollar una determinada actividad o } \\
\text { trabajo, ni la desafiliación para mantenerse en éstos. La ley determinará } \\
\text { las profesiones que requieren grado o título universitario y las } \\
\text { condiciones que deben cumplirse para ejercerlas". Todos los títulos } \\
\text { académicos tienen automáticamente habilitación profesional, con la } \\
\text { sola excepción del título de abogado que es entregado por la Corte } \\
\text { Suprema de Justicia previa una práctica supervisada. }\end{array}$ \\
\hline Colombia & $\begin{array}{l}\text { El ejercicio profesional no es libre sino que el Estado regula en función } \\
\text { del riesgo social de las profesiones y de la protección al consumidor. } \\
\text { La regulación del mercado profesional por la Constitución no recae en } \\
\text { el Gobierno ni en los gobiernos locales sino en el Congreso Nacional } \\
\text { y en leyes Estatutarias establecidas especialmente. La Constitución } \\
\text { establece que las profesiones de riego social pueden ser reguladas más } \\
\text { fuertemente por el legislador. El legislador define por Ley las destrezas } \\
\text { que debe tener el profesional que forman las Universidades. En algunos } \\
\text { casos como la ley } 12029 \text { del } 2008 \text { del ejercicio de la profesión de } \\
\text { abogado, se establecen los lineamientos del currículo de la formación } \\
\text { de estos profesionales. }\end{array}$ \\
\hline
\end{tabular}




\section{Cuadro 1 - La colegialización profesional en América Latina: análisis de casos nacionales}

\begin{tabular}{|c|c|}
\hline Colombia & $\begin{array}{l}\text { La Corte ha establecido que el titulo no es un derecho adquirido y fijó } \\
\text { los requisitos de actualización para el ejercicio profesional en términos } \\
\text { graduales y también limitó el poder de regulación que pudiese ser } \\
\text { excesivo sobre los mercados profesionales por el Congreso. La Corte } \\
\text { estableció que el Estado es quien regula en el ejercicio profesional pero } \\
\text { que tiene potestad de delegación de ese mandato solamente en los } \\
\text { Colegios Profesionales. Los Colegios otorgan la matrícula de ejercicio } \\
\text { profesional, pero no es requisito la afiliación al Colegio respectivo, ya } \\
\text { que las leyes no han establecido la colegiación obligatoria. }\end{array}$ \\
\hline Costa Rica & $\begin{array}{l}\text { Existen por leyes específicas diversas profesiones que tienen } \\
\text { colegiación obligatoria. Los Colegios han creado la Federación de } \\
\text { Colegios Profesionales -FECOPROU- (1966) constituida según Ley de la } \\
\text { República. Actualmente la Federación tiene } 28 \text { colegios profesionales } \\
\text { universitarios afiliados. Varios colegios profesionales han establecido, } \\
\text { en forma voluntaria, mínimos de tarifas de sus profesionales. }\end{array}$ \\
\hline Ecuador & $\begin{array}{l}\text { La ley de educación estableció que la Certificación es un requisito } \\
\text { para ejercer la profesión, para ingresar al Colegio de Profesionales } \\
\text { correspondiente y para legalizar las firmas de títulos por viajes al } \\
\text { exterior. Funciona como un acto de fe pública de que un título ha sido } \\
\text { legalmente conferido por una institución legalmente reconocida. La } \\
\text { Certificación se aplica a los títulos conferidos por Instituciones del } \\
\text { Sistema Nacional de Educación Superior que son los títulos de técnico } \\
\text { superior, tecnólogos, tercero nivel (grado) y cuarto nivel (postgrado), y } \\
\text { se realiza ante el CONESUP (Consejo Nacional de Educación Superior). } \\
\text { Dicha Certificación es obligatoria para los títulos de Pregrado, de } \\
\text { Postgrado realizados en el país y también de los Títulos de Postgrado } \\
\text { otorgados por Universidades Extranjeras, para lo cual a su vez se } \\
\text { requiere también Certificado de Reconocimiento del título otorgado } \\
\text { por una Universidad del país. En el 2008, a pedido del Gobierno, el } \\
\text { Tribunal Constitucional declaró la inconstitucionalidad de los artículos } \\
\text { de las leyes que establecían la afiliación obligatoria a los Colegios } \\
\text { Profesionales, por estar contra de los principios constitucionales de } \\
\text { la libertad de asociación y de libertad de empresa. La Corte Suprema } \\
\text { rechazó la resolución del Tribunal Constitucional y declaró que dicha } \\
\text { norma no se aprobará en el caso de los abogados, los cuales continúan } \\
\text { requiriendo la licencia profesional emitida por el Colegio para el } \\
\text { ejercicio profesional. En el resto de las profesiones no es obligatoria la } \\
\text { Colegiación y el registro se realiza únicamente ante el Conesup. }\end{array}$ \\
\hline El Salvador & $\begin{array}{l}\text { La Ley de Educación Superior, establece que es obligación del Ministerio } \\
\text { de Educación mantener un registro de todos los grados y títulos que las } \\
\text { instituciones de educación superior emitan a sus graduados, la cual se } \\
\text { realiza a través de la Dirección Nacional de Educación Superior. No hay } \\
\text { colegiación obligatoria de los profesionales constitucionalmente, ni por } \\
\text { leyes específicas. Existen diversos colegios profesionales cuya afiliación } \\
\text { y requisitos son establecidos por los propios profesionales actuantes y } \\
\text { sus estatutos privados. }\end{array}$ \\
\hline
\end{tabular}




\title{
Cuadro 1 - La colegialización profesional en América Latina: análisis de casos nacionales
}

\begin{abstract}
Guatemala
La Constitución estableció que la colegiación de los profesionales universitarios es obligatoria. Para el ejercicio de todas las profesiones universitarias es imprescindible tener la calidad de colegiado activo y los Colegios funcionan de conformidad con una Ley de Colegiación Profesional obligatoria que regula el marco general así como de leyes específicas de los diversos colegios. La ley marco establece que los egresados deben cumplir los créditos que cada Colegio establezca. Los Colegios pueden incluir la suspensión definitiva del ejercicio profesional pero sólo cuando medie una sanción judicial.
\end{abstract}

Honduras

México
La Constitución de Honduras establece la Colegiación Profesional obligatoria para el ejercicio de las Profesiones. Los Colegios profesionales son creados por Ley y cada Colegio debe llevar, obligatoriamente, el registro de los colegiados. Si no existe el Colegio respectivo de una profesión, los egresados universitarios se deben afiliar al colegio afín a dicha disciplina. Todos los afiliados están obligados a aportar financieramente a los Colegios. El no pago da derecho a la exclusión de padrón en los colegios y por ende en la pérdida del ejercicio profesional. Los Colegios tienden a fijar los mínimos salariales.

En el país la colegiación no es obligatoria por Ley sino que la Constitución establece la libertad de asociación. El licenciamiento se realiza en los Estados, los cuales han cedido ese rol al Gobierno Federal que lo realiza a través de la Dirección General de Profesiones de la Secretaria de Educación Pública que mantiene el registro nacional y la expedición de una cédula profesional que es el requisito obligatorio para el ejercicio laboral. La Ley General de Profesiones, reglamentaria del artículo 5 Constitucional, que exige registro y obtención de una cédula profesional sólo para el ejercicio de 20 profesiones que son las controladas por el Estado. Ello es un proceso administrativo por parte de la SEP y ante las Direcciones de Profesiones de los gobiernos estatales que así lo requieren, lo cual concluye en una cédula que faculta el ejercicio profesional en el campo donde se ha obtenido el título y de forma vitalicia. La colegiación no es obligatoria en general, pero algunas leyes lo han establecido puntualmente, pero en tanto se están creando diversidad de Colegios de una misma profesión a nivel de los Estados, de hecho existe un sistema de obligación de colegiación pero no de monopolio de Colegios. Algunos Colegios profesionales requieren componentes académicos para el ejercicio profesional y como requisitos para la colegiación, como los Contadores que tienen un examen obligatorio. 


\section{Cuadro 1 - La colegialización profesional en América Latina: análisis de casos nacionales}

\begin{tabular}{|c|c|}
\hline Nicaragua & $\begin{array}{l}\text { La Constitución de Nicaragua establece que "todo nicaragüense tiene } \\
\text { derecho a elegir y ejercer libremente su profesión u oficio y a escoger } \\
\text { un lugar de trabajo, sin más requisitos que el título académico y } \\
\text { que cumpla una función social". La Ley General de Colegiación y del } \\
\text { Ejercicio Profesional (2007) estableció que los Colegios deberán ser } \\
\text { creados por Ley y fija que entre sus cometidos el organizar un registro } \\
\text { profesional, certificar los documentos que consten en su registro, } \\
\text { fundamentalmente el título académico, teniendo dicha certificación } \\
\text { valor de Documento Público. La colegiación no es obligatoria pero } \\
\text { se establecen beneficios y mecanismos que de hecho imponen su } \\
\text { cumplimiento. }\end{array}$ \\
\hline Panamá & $\begin{array}{l}\text { La mayor parte de las profesiones (39) están reglamentadas por } \\
\text { Consejos Técnicos a los cuales les compete expedir la idoneidad } \\
\text { o habilitación de los graduados de las instituciones de educación } \\
\text { superior. }\end{array}$ \\
\hline Paraguay & $\begin{array}{l}\text { La habilitación profesional de los títulos extendidos por las } \\
\text { Universidades la realiza el Ministerio de Educación y Cultura que } \\
\text { tiene un registro para tal efecto. No hay colegiación obligatoria, ni un } \\
\text { sistema de delegación colegial del control matricular y de ejercicio de la } \\
\text { profesión ni fijación salarial profesional. }\end{array}$ \\
\hline Perú & $\begin{array}{l}\text { La Constitución Política del Perú, precisa que los Colegios Profesionales } \\
\text { son instituciones autónomas con personalidad de derecho público } \\
\text { y diversas leyes de profesiones (20) han establecido la colegiación } \\
\text { obligatoria y la función pública de los Colegios en la fiscalización } \\
\text { y control del ejercicio profesional. Los Colegios están altamente } \\
\text { regionalizados a nivel de las Provincias del país y unificados en redes } \\
\text { nacionales y de las diversas especializaciones como monopolios de la } \\
\text { fiscalización del ejercicio de las profesiones. Tales organizaciones se han } \\
\text { unido en el Consejo Nacional de Decanos de los Colegios Profesionales } \\
\text { del Perú, institución autónoma de derecho público interno sin fines } \\
\text { de lucro, reconocido por ley } 28948, \text { que representa a todas las } \\
\text { profesiones organizadas en Colegios Profesionales del país y designa los } \\
\text { profesionales en diversos órganos consultivos del Estado. }\end{array}$ \\
\hline Dominicana & $\begin{array}{l}\text { La Ley establece el requisito de un exequátur otorgado por el Poder } \\
\text { Ejecutivo para el ejercicio en el país de todas las profesiones que exijan } \\
\text { título universitario nacional o extranjero debidamente revalidado, que } \\
\text { se otorga por Decreto en el cual se publica la lista de los profesionales } \\
\text { autorizados a ejercer. Los Títulos y Certificaciones emitidos por las } \\
\text { instituciones de educación superior requieren de una Certificación de } \\
\text { la Secretaria de Estado de Educación Superior, Ciencia y Tecnología } \\
\text { (SEESCYT) como criterio de validación de los mismos. Los Colegios } \\
\text { profesionales son creados por Ley. No existe colegiación obligatoria } \\
\text { generalizada, pero ella se está realizando a partir de leyes específicas } \\
\text { que establecen su obligatoriedad para el ejercicio profesional. }\end{array}$ \\
\hline
\end{tabular}


Cuadro 1 - La colegialización profesional en América Latina: análisis de casos nacionales

(conclusión)

\begin{tabular}{ll}
\hline Uruguay & Los títulos de las Universidades privadas para obtener su validez \\
deben ser registrados ante el Ministerio de Educación y Cultura, con \\
lo cual obtienen idénticos efectos jurídicos que los expedidos por la \\
Universidad de la República. Todos los títulos del área de salud deben \\
registrarse en el Ministerio de Salud Pública (MSP) para su habilitación, \\
y todos los egresados con titulo de abogado y procurador deben \\
prestar juramento ante la Suprema Corte de Justicia, como registro y \\
licenciamiento obligatorio para ejercer ante el Poder Judicial. No hay \\
colegiación obligatoria, ni un sistema de delegación colegial del control \\
matricular y de ejercicio de la profesión y los colegios no actúan en los \\
niveles salariales. \\
No hay ley de Colegiación obligatoria generalizada, pero hay muchas \\
leyes específicas de colegiación obligatoria por profesiones. La Ley \\
de Universidades establece que los títulos y certificados que expiden \\
las Universidades Privadas sólo producirán efectos legales al ser \\
refrendados por el Ejecutivo Nacional. Los títulos de las Universidades \\
públicas no requieren registro. La Constitución establece que “la ley \\
determinará las profesiones que requieren título y las condiciones \\
que deben cumplirse para ejercerlas, incluyendo la colegiación". La \\
colegiación es obligatoria por ley respectiva y no general para todos los \\
profesionales. Los Colegios profesionales se constituyen a nivel de los \\
Estados y se reúnen en Federaciones profesionales.
\end{tabular}

Fuente: RAMA, 2009.

El marco anterior muestra que en la región no es homogéneo el nivel de ingerencia de los Colegios Profesionales en la regulación del ejercicio laboral y el tal sentido tampoco su potestad de regular los niveles salariales. En pocos casos ello se ha dado pero se constata un avance.

En Brasil, algunos colegios profesionales, como los ingenieros y los geólogos, han establecido mínimos salariales para sus colegiados. En Venezuela, por ejemplo, el Colegio de Ingenieros tiene un Tabulador de Sueldos y Salarios Mínimos Profesionales CIV, aprobado por su Junta Directiva Nacional, a ser implementado todos los años, en el cual se determina el Salario Mínimo neto, de acuerdo al Nivel Profesional y donde no se incluyen los beneficios de Ley, ni los beneficios contractuales u otros.

En Honduras, como en Guatemala, la Constitución establece la colegialización obligatoria de los profesionales universitarios. En este 
contexto los diversos colegios han ido estableciendo estatutos de funcionamiento y de regulación, entre los cuales la existencia del salario mínimo que deberían recibir los profesionales del Colegio respectivo. Ello derivó en diversos niveles de salarios mínimos por profesiones, los cuales se fijaron en forma nominal. Sin embargo la inflación, devaluación y no actualización derivo en una caída relativa de ellos, lo cual derivó en el establecimiento de dichos salarios como un múltiplo del salario mínimo nacional de los trabajadores no profesionales. Ello llevó a diversos múltiplos como se detalla en la Tabla 4.

Tabla 4 - Salarios mínimos de distintos profesionales en diversos años (Honduras)

\begin{tabular}{lcc}
\hline Año & Profesión & Salarios mínimos (2006) \\
\hline 1999 & Enfermeras profesionales & 4 \\
1994 & Químicos Farmacéuticos & 4 \\
1994 & Químicos Farmacéuticos con & 5 \\
& maestría & 5 \\
1993 & Cirujano dentista & 1.2 \\
1994 & Cirujano dentista especialista & 1 \\
1997 & Trabajador social & 2 \\
1997 & Microbiólogos y químicos & 3 \\
1997 & Microbiólogos y químicos & especialistas \\
1985 & Médicos & 12 \\
1997 & Médicos & 14 \\
1997 & Médicos especialistas & \\
\hline
\end{tabular}

Fuente: SALARIOS, 2014.

Los casos son crecientes y están dados por la acción de los colegios profesionales. En los países donde la colegiación es obligatoria, el avance en esta dirección es más marcado. 
A la vez se manifiestan avances hacia regulaciones nacionales. En Colombia por ejemplo se presentó un proyecto de ley que definió el salario mínimo asignado a los profesionales universitarios en cualquier campo de las ciencias o las artes, teniendo como base de su cálculo el salario mínimo legal mensual de cada año establecido por el Gobierno Nacional. Este salario mínimo profesional se propuso como equivalente a dos y medio salarios mínimos legales mensuales vigentes de cada año. El proyecto no fue aprobado. Dada la existencia de alta diversidad de salarios mínimos de trabajadores, es dable igualmente visualizar la alta diversidad de los salarios profesionales en toda la región. En Costa Rica, por su parte, el salario mínimo incluye el nivel profesional. El Ministerio de Trabajo emite un Decreto semestral de los salarios mínimos nacionales para el sector privado con cientos de puestos de trabajo y entre los cuales incluye los técnicos de educación superior, los diplomados de educación superior, los bachilleres universitarios y los licenciados universitarios ${ }^{2}$.

\section{Conclusiones}

El aumento de los egresos universitarios está cambiando los mercados profesionales. Ello está expresándose en un lento inicio de fijación de salario mínimo profesional. Estas iniciativas son derivadas de cambios en la tasa de retornos ante la tendencia a la sobreoferta profesional. En varios mercados se constata que los Colegios profesionales están estableciendo políticas de fijación salarial. Tal situación se diferencia según los marcos normativos de la regulación del ejercicio profesional en los diversos países. En ello incide fuertemente además el carácter obligatorio o no de la colegialización, y si ello es monopólico por parte de un solo colegio profesional. Más allá de las regulaciones a nivel de los Colegios Profesionales se constata que en la fijación del

2 Disponible en: <https://docs.google.com/viewer?a=v\&pid=sites\&srcid=bXRzcy5nby5jcnxtdHNzfG d4OjRIO GU1YzUwMjliNzZkNzE>. Accedido el: 20 jul. 2015. 
salario mínimo hay fuertes diferenciaciones por tipos de trabajo y entre ellos se comienza a visualizar aquellos del sector terciario. En Costa Rica la regulación del salario mínimo privado a la vez incluye un mínimo universitario, desde el cual los colegios pueden establecer sus propios niveles mínimos para los distintos profesionales.

El nuevo contexto de recomposición de los mercados profesionales con una tendencia estructural al aumento de la oferta de profesionales en forma superior a la demanda de trabajo especializado, deriva en diversos impactos en los mercados laborales, entre los cuales el aumento del desempleo y la emigración, y también en la fijación de las remuneraciones profesionales. Esta se expresa en forma aislada en acciones de algunos colegios profesionales y en algunos países, pero de hecho pudiera constituirse en un componente de la política pública a futuro. Actualmente la política pública se focaliza en la expansión de la oferta a través de la gratuidad estimulando la formación de recursos humanos, en un contexto donde las tasas de retornos son superiores respecto a los retornos de las personas sin formación terciaria. Los niveles de desempleo, los tiempos de desempleo antes del ingreso a los mercados de trabajo y la propia tasa de retorno, han impulsado a las personas a una creciente demanda educativa con miras a alcanzar esos posicionamientos en los mercados de trabajo como resultado de la adquisición de capacidades y competencias superiores. Sin embargo, al aumentar los egresos, tender a caer los retornos salariales, se produce una mayor presión hacia una mayor gratuidad educativo, lo cual a su vez renueva la problemática de mayores egresos y más caídas de las tasas de retornos y de los salarios profesionales. En esta dinámica en construcción como derivación de la masificación el sector privado será el sector más afectado, constatándose en la región ve situaciones donde la matrícula se acerca al salario de enganche inicial de los profesionales.

El sistemático incremento del desempleo profesional en América Latina y la caída de los retornos, como resultado de las nuevas dinámicas de los mercados de oferta y demanda profesional, puede ser analizada desde el lado de una escasa demanda de trabajo profesional o desde el enfoque que planteamos desde el lado de la existencia de una 
sobreoferta, pero bajo ambas miradas ello se expresará en cambios en los niveles de salarios, en el desempleo y en la emigración profesional. En la región históricamente han existido bajos niveles de desempleo profesionales y salarios elevados como resultado de ese desequilibrio en términos de la oferta de egresados y de menor demanda de profesionales, más allá de las competencias. Esos salarios profesionales expresaban dos componentes: por un lado el capital humano adquirido en términos de aporte a la productividad, y por el otro lado los niveles de equilibrios de oferta y demanda de trabajo. Este segundo componente es el que está cambiando y el aumento del egreso impacta en la relación salario productividad y reduce los retornos de los egresados. En algunos mercados ello se expresa en una mayor diferencia entre los salarios de los egresados de los programas acreditados y una mayor prima por los estudios de postgrado. Pero más allá de estas dinámicas, el impacto del mayor egreso que la demanda finalmente deteriorará el salario de los profesionales a nivel del grado desestimulando el sacrificio de estudiar. El inicio de políticas de fijación de salarios mínimos profesionales marca una forma de resolver el problema con miras a mantener el incremento de la cobertura y de los egresos, mostrando que el salario mínimo profesional estará en la agenda universitaria a futuro con creciente intensidad.

\section{Referencias}

ADROGUÉ, C. Desempleo y retornos de la educación superior en Argentina (19742002). Buenos Aires: IAE-UCEMA, 2006.

ARAGONÉS, A. M. (Coord.). Mercados de trabajo y migración internacional. México: UNAM, Instituto de Investigaciones Económicas, 2011.

ARAGONÉS, A. M.; SALGADO, U. Mercados del trabajo en la economía del conocimiento y el fenómeno migratorio. El caso de Estados Unidos (1990-2006). In. ARAGONÉS, A. M. (Coord.). Mercados de trabajo y migración internacional. México: UNAM, Instituto de Investigaciones Económicas, 2011. p. 79-118. 
CARNOY, M. La economía de la educación. Barcelona: UOC, 2006.

COMISIÓN ECONÓMICA PARA AMÉRICA LATINA Y EL CARIBE - CEPAL; ORGANIZACIÓN INTERNACIONAL DEL TRABAJO - OIT. Protección social universal en mercados laborales con informalidad. Coyuntura laboral en América Latina y el Caribe, n. 12, Mayo 2015. Disponible en: <http://repositorio.cepal. org/bitstream/handle/11362/38051/S1500311_es.pdf?sequence=1〉. Accedido el: 20 jul. 2015.

FORERO, N.; RAMIREZ, M. Determinantes de los ingresos laborales de los graduados universitarios durante el período 2001-2004. Bogotá: Universidad de Rosario, 2008.

HOPER EDUCAÇÃO. Análise setorial do ensino superior privado: Brasil 2014. Foz do Iguaçu: Hoper Educação, 2014.

ORGANIZACIÓN INTERNACIONAL DEL TRABAJO - OIT. Informe sobre el trabajo en el mundo 2012: mejores empleos para una economía mejor. Madrid, 2013. ORGANIZACIÓN PARA LA COOPERACIÓN Y EL DESARROLLO ECONÓMICOS OCDE. La educación superior hacia el 2030. México: Instituto Internacional de Investigación en Tecnología Educativa, 2012.

PSACHAROPOULOS, G. Higher education in developing countries: a cost-benefit analysis . Washington: World Bank, 1980.

PSACHAROPOULOS, G. The economic impact of education. Washington: World Bank, 1994

RAMA, C. Los postgrados de América Latina y el Caribe en la sociedad del conocimiento. México: Udual, 2007.

RAMA, C. Paradigmas emergentes, competencias profesionales y nuevos modelos universitarios en América Latina. Santo Domingo: Unicaribe, 2009 
RAMA, C. Nuevas dinámicas de los mercados laborales y educativos de profesionales con la masificación de la cobertura y la expansión del egreso. In: ARAGONES, A.M. (Coord.). Crisis económica y migración ¿Impactos temporales o estructurales? México: UNAM, Instituto de Investigaciones Económicas, 2014. p. 201-236.

RED DE INDICADORES DE CIENCIA Y TECNOLOGÍA IBEROAMERICANA E INTERAMERICANA - RICYT. Graduados en educación superior. 2015. Disponible en: $\quad$ http://db.ricyt.org/query/AR,BO,BR,CA,CL,CO,CR,CU,EC,ES,GT,HN,J M,MX,NI,PA,PE,PR,PT,PY,SV,TT,US,UY,VE,AL,IB/1990\%2C2012/CGRADO>. Accedido el: 20 jul. 2015.

SALARIOS mínimos de los profesionales universitarios en Honduras. In: Wikipedia. 2014. Disponible en: <https://es.wikipedia.org/wiki/ Anexo:Salarios_m\%C3\%ADnimos_de_los_profesionales_universitarios_en_ Honduras>. Accedido el: 20 jul. 2015.

VARELA, R.; URCIAGA, J. Diferencias salariales en México: una perspectiva de educación y actividad económica. Revista de Educación Superior, v. 41, n. 162, p. 25-43, 2012.

VRIES, W.; NAVARRO, Y. Profesionistas del futuro o futuro taxistas? Los egresados universitarios y el mercado laboral en México. Revista Iberoamericana de Educación Superior, v. 2, n. 4, p. 2-27, 2011.

WELLER, J. El empleo terciario en América Latina: entre la modernidad y la sobrevivencia. Revista de la CEPAL, v. 84, p. 159-175, 2004.

Recibido: 05/09/2014

Received: 09/05/2014

Aprobado: 10/12/2014

Approved: 12/10/2014 